\title{
The Characterizations of Discrete Life Distribution Class with Relation to Geometric Distribution
}

\author{
Ronghua Wang ${ }^{1}$, Naijun Sha ${ }^{2}$, Beiqing $G u^{3}$ and Xiaoling $X u^{4}$ \\ ${ }^{1}$ College of Mathematics and Science, Shanghai Normal University, Shanghai 200234, China \\ ${ }^{2}$ Department of Mathematical Sciences, University of Texas at El Paso, TX 79968, USA \\ ${ }^{3}$ Business Information Management School, Shanghai Institute of Foreign Trade, Shanghai 201600, China \\ ${ }^{4}$ Business Information Management School, Shanghai Institute of Foreign Trade, Shanghai 201600, China
}

Received: 7 Feb. 2013, Revised: 16 Feb. 2013, Accepted: 19 Mar. 2013

Published online: 1 Jul. 2013

\begin{abstract}
In this paper, we present some characterizations of discrete life distributions, especially for dHNBUE, dHNWUE, dNBUE and dNWUE classes, and their relations with geometric distribution. We characterize the geometric distribution through the ordered binomial moment and study the approximation between some discrete life classes and geometric distribution via an upper bound of the probability difference. The upper bounds presented are more rigorous than the ones given previously.
\end{abstract}

Keywords: Discrete life distribution; Geometric distribution; Binomial moment; Factorial moment; Upper bound of probability difference.

\section{Introduction}

In time-to-event studies, discrete lifetimes arise in various common situations where either the measurements of time are often slotted or occurred events are recorded by fixed length intervals or measured discretely, such as inserting or joining products. In other reliability testings, often times the tested units cannot be monitored continuously, but instead be inspected at constant periods, so their failure times can also be treated as discrete random variables. The most common discrete lift distribution is the geometric, the analog of the exponential distribution for continuous lifetimes. Because of the memoryless property, geometric distribution is a basic component of different kinds of stochastic models and plays an essential role in reliability theory and applied probabilistic models [3,4,14]. However, the geometric imposes limitation on its use, thus other or general discrete life distributions have been discussed and applied in various situations, e.g. [1,17,19] and the references therein. Alternatively, the study of the relation among life distributions becomes a focus in recent years, concentrating mainly on the approximation among various kinds of life distribution classes (or other special distributions) describing random phenomena like aging and wearing from different perspectives [5,21]. Much work has been focused on continuous life distribution classes, see [6] for a systematic treatment of this topic. There has been relatively less work done among discrete life distribution classes, except the work in Cheng [6], who studied the approximation among the dNBUE, dDMRL, dIMRL, dDFR and dNWUE classes. Especially, the research on the relation between the geometric and other discrete life distributions is only at the very beginning. In this article, we focus on the properties of various discrete life distributions, particularly for the dHNBUE, dHNWUE, dNBUE and dNWUE classes, and the approximation by the geometric. The rest of the paper is organized below. In Section 2 we present the definitions and characteristics for various discrete life distributions, and their relations with geometric distribution. We study the upper bounds to approximate dNBUE and dDMRL on the geometric distribution in Section 3. The upper bounds improves the ones given in [12]. We conclude the article with a brief discussion in Section 4.

\footnotetext{
${ }^{*}$ Corresponding author e-mail:
} 


\section{The Characteristics of Discrete Life Distribution Class}

Before presenting some important discrete life distributions, we first define some common functions and notations to be used throughout the paper. For the purpose of notation simplicity and clear presentation, we denote $\mathscr{H}=\{X$ : a nonnegative integer valued random variable with finite mean $\mu\}$, the sets of nonnegative integers $\mathscr{N}=\{0,1,2, \cdots\}$ and positive integers $\mathscr{N}_{+}=\{1,2, \ldots\}$. Suppose that $X \in \mathscr{H}$ follows a discrete life distribution $p(k)=P(X=k)$ with domain $\mathscr{N}$, then the other functions are usually defined as

(1) Distribution function $F(k)=P(X<k)=\sum_{i=0}^{k-1} p(i)$ with $\sum_{i=0}^{-1}=0$;
(2) Survival function $\bar{F}(k)=1-F(k)=P(X \geq k)=\sum_{i=k}^{\infty} p(i)$;
(3) Failure rate function $\lambda(k)=P(X=k \mid X \geq k)=\frac{p(k)}{\bar{F}(k)}$;
(4) The mean life $\mu=E X=\sum_{k=0}^{\infty} k p(k)=\sum_{k=1}^{\infty} \bar{F}(k)$;
(5) The mean reliability function $\bar{G}(k)=\frac{1}{1+\mu} \sum_{j=k}^{\infty} \bar{F}(j)$ for $\mu<\infty$.

(6) The mean remaining life $\mu(k)=E(X-k \mid X \geq k)+1=\frac{\sum_{j=k}^{\infty} \bar{F}(j)}{\bar{F}(k)}=\frac{(1+\mu) \bar{G}(k)}{\bar{F}(k)}$.

In probability and statistics, the moments of a random variable serve as useful metrics that provide a significant amount of information about a distribution. Rather than expressing the moments by the probability function, we introduce another useful expression, in which the moment of $X$ is represented by the survival function.

Lemma 1. For $X \in \mathscr{H}$, the rth moment $E X^{r}=\sum_{k=1}^{\infty}\left[k^{r}-(k-1)^{r}\right] \bar{F}(k), r \in \mathscr{N}$.

Proof.For $r \in \mathscr{N}$, we simply have

$$
\begin{aligned}
E X^{r} & =\sum_{k=0}^{\infty} k^{r} P(X=k)=\sum_{k=0}^{\infty} k^{r}[\bar{F}(k)-\bar{F}(k+1)] \\
& =\sum_{k=1}^{\infty} k^{r} \bar{F}(k)-\sum_{k=1}^{\infty}(k-1)^{r} \bar{F}(k)=\sum_{k=1}^{\infty}\left[k^{r}-(k-1)^{r}\right] \bar{F}(k)
\end{aligned}
$$

In particular, the first two moments are $\mu=E X=\sum_{k=1}^{\infty} \bar{F}(k)$ and $E X^{2}=\sum_{k=1}^{\infty}(2 k-1) \bar{F}(k)$.

In the last few decades, many discrete life distributions were proposed for modeling life data on various occasions. Cheng [6] summarized the classes of some common discrete life distributions in the followings.

Definition 1. Let $X \in \mathscr{H}$, then it belongs to

a) discrete increasing (decreasing) failure rate dIFR (dDFR) class if $\lambda(k)$ is increasing (decreasing) function for $k \in \mathscr{N}$.

b) discrete increasing (decreasing) failure rate average dIFRA (dDFRA) class if $\bar{F}^{\frac{1}{k}}(k)$ is decreasing (increasing) for $k \in \mathscr{N}$.

c) discrete increasing (decreasing) mean-residual-life dIMRL (dDMRL) class if $\mu(k)$ is increasing (decreasing) for $k \in \mathscr{N}$.

d) discrete new-better(worse)-than-used dNBU (dNWU) class if $P(X-k \geq j \mid X \geq k) \leq P(X \geq j)$, i.e. $\bar{F}(k+j) \leq$ $\bar{F}(k) \bar{F}(j)(\bar{F}(k+j) \geq \bar{F}(k) \bar{F}(j))$ for $k, j \in \mathscr{N}$.

e) discrete new-better(worse)-than-used-expectationdNBUE (dNWUE) class if $E(X-k \mid X \geq k) \leq E X=\mu$, i.e. $\bar{G}(k) \leq$ $\bar{F}(k)(\bar{G}(k) \geq \bar{F}(k))$ for $k \in \mathscr{N}$.

f) discrete harmonically new-better (worse)-than-used-expectation dHNBUE (dHNWUE) class if $\bar{G}(k) \leq\left(\frac{\mu}{1+\mu}\right)^{k}\left(\bar{G}(k) \geq\left(\frac{\mu}{1+\mu}\right)^{k}\right), k \in \mathscr{N}$

g) discrete $L d L(d \bar{L})$ class if $\sum_{k=0}^{\infty} \bar{F}(k) s^{k} \leq \frac{1+\mu}{1+(1-s) \mu}\left(\sum_{k=0}^{\infty} \bar{F}(k) s^{k} \geq \frac{1+\mu}{1+(1-s) \mu}\right)$ for $0<s \leq 1$.

The inclusion relations among the above-mentioned classes are as follows 


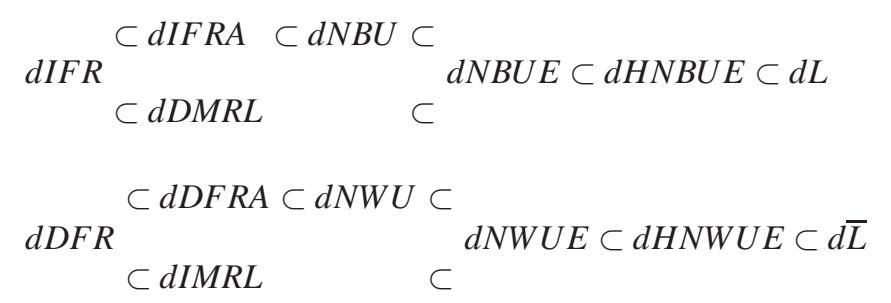

Specifically, for the geometric random variable $X \sim G e o(p)$ with probability function $P(X=k)=p q^{k}, k \in \mathscr{N}$, where the probabilities of success and failure on each trial are $p$ and $q=1-p$, we have $\mu=E X=\frac{q}{p}$, and so $p=\frac{1}{1+\mu}$ and $q=\frac{\mu}{1+\mu}$ (hence it can also be written as $X \sim G e o\left(\frac{1}{1+\mu}\right)$ ). In addition, $\bar{F}(k)=P(X \geq k)=q^{k}=\left(\frac{\mu}{1+\mu}\right)^{k}, \bar{G}(k)=\left(\frac{\mu}{1+\mu}\right)^{k}, \lambda(k)=$ $p=\frac{1}{1+\mu}, \mu(k)=1+\mu, j, k \in \mathscr{N}$. Clearly, the geometric distribution belongs to all the above life distribution classes.

To explore the relation between the geometric and other discrete life distributions, we first introduce some definitions and notations.

Definition 2. The rth ascending factorial moment of the random variable $X$ about the point $b$ is defined as

$\mu_{(r)}(b)=E\left[(X-b)_{(r)}\right]=E[(X+r-1-b)(X+r-2-b) \cdots(X-b)]$

Particularly, when $b=0, \mu_{(r)}(0)$ is called the rth ascending factorial moment, written as $\mu_{(r)}$; and when $b=E X=$ $\mu, \mu_{(r)}(\mu)$ is called the rth ascending central moment, written as $\mu_{(r)}^{\prime}$.

By using the finite difference method, Fang and Xu [13] derived the ascending factorial moment for the geometric distribution.

Lemma 2. If $X \sim G e o(p)$ with $P(X=k)=p q^{k}, k \in \mathscr{N}$, then the rth ascending factorial moment

$\mu_{(r)}^{g}=E[(X+r-1)(X+r-2) \cdots(X+1) X]=r ! \frac{q}{p^{r}}=r ! \mu(1+\mu)^{r-1}, r \in \mathscr{N}_{+}$.

Related to the ascending factorial moment, other commonly used moments and the characteristic index for a distribution were defined as follows [20].

Definition 3. For $X \in \mathscr{H}$, define

(1) the rth order binomial moment $\beta_{r}=E\left(\begin{array}{c}X+r \\ r\end{array}\right)=\sum_{k=0}^{\infty}\left(\begin{array}{c}k+r \\ r\end{array}\right) P(X=k)$;

(2) the rth characteristic index number $\alpha_{r}=\left|1-\frac{\beta_{r}}{(1+\mu)^{r}}\right|, r \in \mathscr{N}$.

Obviously, $\beta_{r}=\frac{1}{r !} \mu_{(r)}(-1)$, and the first binomial moment $\beta_{1}=1+\mu$ and characteristic index $\alpha_{1}=0$.

For $X \sim G e o\left(\frac{1}{1+\mu}\right)$, by Lemma 2 , it is easily seen the recursive form $\beta_{r}-\beta_{r-1}=\frac{q}{p^{r}}$ with $\beta_{0}=1$, hence $\beta_{r}=$ $\frac{1}{p^{r}}=(1+\mu)^{r}$, and then $\alpha_{r}=0, r \in \mathscr{N}$. Notice that the $\alpha_{2}$ proportional to the $\alpha$ (up to a constant) defined in [6] (actually $\alpha_{2}=\left(\frac{\mu}{1+\mu}\right)^{2} \alpha$ ), who pointed out that $\alpha_{2}=0$ cannot guarantee the random variable $X$ following the geometric distribution. However, if confining the study to the dHNBUE or dHNWUE class, Cheng [6] concluded that $\alpha_{2}=0$ will be able to characterize the geometric distribution (thereafter $\alpha_{r}=0, r \in \mathscr{N}$ ). We may extend to the fact that in dHNBUE or dHNWUE class, $\alpha_{r}=0$ for some integer value $r \geq 2$ (not necessarily $\alpha_{2}=0$ ) will leads to the geometric distribution. Before proving this result, we first present some features for dHNBUE and dHNWUE classes, whose detail proofs were provided in the Appendix.

Lemma 3. For $X \in d H N B U E$, we have $\beta_{r} \leq \beta_{r}^{g}$ and $\mu_{(r)} \leq \mu_{(r)}^{g}, r \in \mathscr{N}$, where $\beta_{r}^{g}=(1+\mu)^{r}$ and $\mu_{(r)}^{g}=r ! \mu(1+\mu)^{r-1}$ are the rth order binomial and ascending factorial moments for Geo $\left(\frac{1}{1+\mu}\right)$, and so $\alpha_{r}=1-\frac{\beta_{r}}{(1+\mu)^{r}}$. Alternatively, for $X \in$ $d H N W U E$, then $\beta_{r} \geq \beta_{r}^{g}$ and $\mu_{(r)} \geq \mu_{(r)}^{g}$, and so $\alpha_{r}=\frac{\beta_{r}}{(1+\mu)^{r}}-1, r \in \mathscr{N}$.

Lemma 4. (1) If $X \in d H N B U E$ or $d H N W U E$, then $\alpha_{r} \geq \frac{\alpha_{r-1}}{1+\mu}$. In addition, for $X \in d H N B U E$, we have $\alpha_{r}=1-$ $\frac{\beta_{r}}{(1+\mu)^{r}}<1-\frac{1}{r !}, r \geq 2$. (2) If $X \in d N B U E$ or $d N W U E$, then $\alpha_{r} \geq \alpha_{r-1}, r \geq 2$. 
Now we may prove the following theory about the characterization of the geometric distribution.

Theorem 1. If $X \in d H N B U E$ or $d H N W U E$ with finite mean $\mu$, then $X \sim G e o\left(\frac{1}{1+\mu}\right)$ if and only if $\alpha_{r}=0$ for some integer value $r \geq 2$.

Proof. Since $\alpha_{r}=0$ for any $r \geq 2$ for $G e o\left(\frac{1}{1+\mu}\right)$, we only need prove the necessity. We have $\beta_{r}=(1+\mu)^{r}$ from $\alpha_{r}=0$, and so $\bar{G}(k)=\left(\frac{\mu}{1+\mu}\right)^{k}, k=0,1,2, \ldots$ from the proof of Lemma 3. Thus $\bar{F}(k)=(1+\mu)[\bar{G}(k)-\bar{G}(k+1)]=\left(\frac{\mu}{1+\mu}\right)^{k}$, and then $P(X=k)=\bar{F}(k)-\bar{F}(k+1)=\left(\frac{1}{1+\mu}\right)\left(\frac{\mu}{1+\mu}\right)^{k}, k \in \mathscr{N}$, hence $X \sim G e o\left(\frac{1}{1+\mu}\right)$.

The theorem indicates that for dHNBUE or dHNWUE class, any one $\alpha_{r}=0, r \geq 2$ leads to all $\alpha_{r}=0, r=2,3, \ldots$. However, this is not true for other discrete life distribution classes. The following example demonstrates that generally $\alpha_{2}=0$ and $\alpha_{3}=0$ are not equivalent each other.

Example 1. $\alpha_{2}=0$ but $\alpha_{3} \neq 0$.

Suppose the discrete random variable $X$ has possible values 0,2 with probabilities $\frac{3}{4}, \frac{1}{4}$, respectively. Thus $\mu=\frac{1}{2}$, and $\bar{G}(2)=\frac{1}{1+\mu} \sum_{j=2}^{\infty} \bar{F}(j)=\frac{1}{1+\mu} \bar{F}(2)=\frac{1}{6}>\left(\frac{\mu}{1+\mu}\right)^{2}=\frac{1}{9}$, but $\bar{G}(3)=\frac{1}{1+\mu} \sum_{j=3}^{\infty} \bar{F}(j)=0<\left(\frac{\mu}{1+\mu}\right)^{3}=\frac{1}{27}$. Hence $X \notin \mathrm{dHNBUE}$ or dHNWUE, and we have $\beta_{2}=\frac{1}{2} E[(X+2)(X+1)]=\frac{9}{4}, \alpha_{2}=\left|1-\frac{9 / 4}{(1+1 / 2)^{2}}\right|=0$ and $\beta_{3}=\frac{1}{6} E[(X+3)(X+2)(X+1)]=$ $\frac{13}{4}, \alpha_{3}=\left|1-\frac{13 / 4}{(1+1 / 2)^{3}}\right|=\frac{1}{27} \neq 0$.

In the next section, we explore the difference between discrete life distribution and geometric distribution.

\section{Approximating Discrete Life Distributions with Geometric Distribution}

There were many researches studying the difference among the distributions, but most works were for the purpose of the hypothesis testing, such as goodness of fit tests [18]. In probability theory, the Kullback-Leibler divergence [16], a relative entropy, was often used as a measure of the difference between two probability distributions. Here we consider the "reliability difference" [7], in which the research of approximations and upper bounds has been studied among various discrete life distribution classes [8-10], and between geometric and some discrete life distribution classes such as dDMRL, dNWUE, dIMRL and dDFR [11,12]. We focus on the approximation between the geometric and other discrete life distributions, such as dNBUE and dDMRL classes. The difference definition is the following.

Definition 4. Suppose $X, Y \in \mathscr{H}$ with same mean $\mu$, the difference of reliabilities is defined as $\Delta(X, Y)=\sup _{k \in \mathscr{N}}|P(X \geq k)-P(Y \geq k)|$.

Particularly, the difference between $X \in \mathrm{dNBUE}$ or dDMRL and $Y \sim G e o\left(\frac{1}{1+\mu}\right)$ is our interest. Cheng and Ma [12] presented 'big' upper bounds of $\Delta(X, Y)$, and we will provide tight upper bounds to improve the approximation. To examine the comparison among various discrete life distributions with the geometric distribution, Cheng and Ma [12] proposed the following measurements.

Definition 5. The following notations are defined as

$A(k)=\bar{F}(k)-\bar{G}(k), \eta(k)=\left(\frac{\mu}{1+\mu}\right)^{k}-\bar{G}(k)$

$\delta(k)=\bar{F}(k)-\left(\frac{\mu}{1+\mu}\right)^{k}=A(k)-\eta(k), k \in \mathscr{N}$.

For the geometric random variable with mean $\mu, \bar{F}(k)=\bar{G}(k)=\left(\frac{\mu}{1+\mu}\right)^{k}$, then clearly, $A(k)=\eta(k)=\delta(k)=0, k \in \mathscr{N}$. In addition, dNBUE (dNWUE) class corresponds to $A(k) \geq 0(A(k) \leq 0)$, and dHNBUE (dHNWUE) class to $\eta(k) \geq$ $0(\eta(k) \leq 0), k \in \mathscr{N}$. Here are some useful preliminary results in $[7,12]$.

Lemma 5. For $X \in \mathscr{H}$, we have

$$
\begin{aligned}
& \text { (1) } \eta(k)=\frac{1}{\mu} \sum_{i=0}^{k-1}\left(\frac{\mu}{1+\mu}\right)^{k-i} A(i) \text {, so that } \delta(k)=A(k)-\frac{1}{\mu} \sum_{i=0}^{k-1}\left(\frac{\mu}{1+\mu}\right)^{k-i} A(i) \text {; } \\
& \text { (2) } A(k) \leq \frac{k}{1+\mu}, k \in \mathscr{N} ; \\
& \text { (3) } A(i) \geq A(k)-\frac{k-i}{1+\mu}, 0 \leq i \leq k .
\end{aligned}
$$

Now we discuss the approximation of dNBUE and dDMRL classes by geometric distribution. 


\subsection{The dNBUE Class}

First, we briefly present the upper bound given in [12]. The following results play a key role in their work.

Lemma 6. 1. If $X \in \operatorname{dHNBUE}(\eta(k) \geq 0, k \in \mathscr{N})$, then $\sum_{k=0}^{\infty} A(k)=\sum_{k=0}^{\infty} \eta(k)=(1+\mu) \alpha_{2}$.

2. If $X \in d N B U E$, then (1) $|\delta(k)| \leq A(k) \leq \sqrt{\frac{2 \mu \alpha_{2}}{1+\mu}} \leq \frac{\mu}{1+\mu} ;(2)-\alpha_{2} \leq \delta(k) \leq 1-\exp \left\{-\frac{1+\mu}{\mu} A(k)\right\}, k \in \mathscr{N}$.

We also observed the following general result.

Lemma 7. For $\mu>0$ and $0 \leq x<\frac{1}{2}$, we have $x \leq 1-\exp \left\{-\sqrt{\frac{2(1+\mu) x}{\mu}}\right\}$.

Proof. It is equivalent to show that $f(x)=\log (1-x)+\sqrt{\frac{2(1+\mu) x}{\mu}} \geq 0$ in $0 \leq x<\frac{1}{2}, \mu>0$. Since $f(0)=0, f\left(\frac{1}{2}\right)=-\log 2+$ $\sqrt{\frac{1+\mu}{\mu}}>-\log 2+1>0$, and $f^{\prime}(x)=\frac{-\sqrt{x}+\sqrt{\frac{1+\mu}{2 \mu}}(1-x)}{\sqrt{x}(1-x)}$. Let $g(x)=-\sqrt{x}+\sqrt{\frac{1+\mu}{2 \mu}}(1-x)$, then $g^{\prime}(x)=-\frac{1}{2 \sqrt{x}}-\sqrt{\frac{1+\mu}{2 \mu}}<0$ and $g(0)=\sqrt{\frac{1+\mu}{2 \mu}}>0$. As a result, the unique root of $g(x)$ is $x_{0}=\frac{1+2 \mu-\sqrt{3 \mu^{2}+2 \mu}}{1+\mu}>\frac{1}{2}$. Thus for $0 \leq x<x_{0}$, we have $g(x)>0$, thus $f^{\prime}(x)>0$. Hence $f(x) \geq 0$ in $0 \leq x<\frac{1}{2}<x_{0}$.

For dHNBUE ( $\supset$ dNBUE) class, we know $0 \leq \alpha_{2}<\frac{1}{2}$ from Lemma 4 , and so it results in $\alpha_{2} \leq 1-\exp \left\{-\sqrt{\frac{2(1+\mu) \alpha_{2}}{\mu}}\right\}$ by Lemma 7. Thus it is straightforward by Lemma 6 to have the following upper bound in [12]

$$
\begin{aligned}
\Delta(X, Y) & =\sup _{k \in \mathscr{N}}|\delta(k)| \leq \max \left\{\alpha_{2}, 1-\exp \left\{-\frac{1+\mu}{\mu} A(k)\right\}\right\} \\
& \leq \max \left\{\alpha_{2}, 1-\exp \left\{-\sqrt{\frac{(1+\mu) \alpha_{2}}{\mu}}\right\}\right\} \\
& \leq 1-\exp \left\{-\sqrt{\frac{2(1+\mu) \alpha_{2}}{\mu}}\right\}
\end{aligned}
$$

In what follows, we provide a tight upper bound for the difference. Suppose that $X \in \mathrm{dNBUE}$ ( $\subset$ dHNBUE) with finite mean $\mu$, then $A(k) \geq 0$ and $\sum_{k=0}^{\infty} A(k)=(1+\mu) \alpha_{2}$ from Lemma 5. Hence $A(k)$ is bounded and we let $A=\sup _{k \in \mathscr{N}} A(k)$. Obviously, the geometric distribution $\left(\alpha_{r}=0\right)$ is equivalent to $A=0$. The following preliminary results (shown in the Appendix) will be used for our improved upper bound in Theorem 2.

Lemma 8. Suppose $X \in d N B U E$ and let $A_{0}=\frac{-1+\sqrt{1+8 \alpha_{2}(1+\mu)^{2}}}{2(1+\mu)}$, then we have (1) $A(k) \leq A \leq A_{0} \leq \sqrt{\frac{2 \mu \alpha_{2}}{1+\mu}} \leq \frac{\mu}{1+\mu}$; $\alpha_{2} \leq 1-\exp \left\{-\frac{1+\mu}{\mu} A_{0}\right\}$.

Theorem 2. Suppose that $X \in d N B U E$ and let $A_{0}=\frac{-1+\sqrt{1+8 \alpha_{2}(1+\mu)^{2}}}{2(1+\mu)}$. For $Y \sim$ Geo $\left(\frac{1}{1+\mu}\right)$, we have

$\Delta(X, Y) \leq 1-\exp \left\{-\frac{1+\mu}{\mu} A_{0}\right\} \leq 1-\exp \left\{-\sqrt{\frac{2(1+\mu) \alpha_{2}}{\mu}}\right\}$

Proof. For $X \in \mathrm{dNBUE}$, by Lemmas 6 and 8, then

$$
\begin{aligned}
-\alpha_{2} & \leq \delta(k) \leq 1-\exp \left\{-\frac{1+\mu}{\mu} A(k)\right\} \\
& \leq 1-\exp \left\{-\frac{1+\mu}{\mu} A_{0}\right\} \leq 1-\exp \left\{-\sqrt{\frac{2(1+\mu) \alpha_{2}}{\mu}}\right\}
\end{aligned}
$$


Secondly, for $Y \sim G e o\left(\frac{1}{1+\mu}\right)$, then $P(Y \geq k)=\left(\frac{\mu}{1+\mu}\right)^{k}$, and so

$$
\begin{aligned}
\Delta(X, Y) & =\sup _{k \in N}\left|\bar{F}(k)-\left(\frac{\mu}{1+\mu}\right)^{k}\right|=\sup _{k \in N}|\delta(k)| \\
& \leq \max \left\{\alpha_{2}, 1-\exp \left\{-\frac{1+\mu}{\mu} A_{0}\right\}\right\} \\
& =1-\exp \left\{-\frac{1+\mu}{\mu} A_{0}\right\}
\end{aligned}
$$

hence overall $\Delta(X, Y) \leq 1-\exp \left\{-\frac{1+\mu}{\mu} A_{0}\right\} \leq 1-\exp \left\{-\sqrt{\frac{2(1+\mu) \alpha_{2}}{\mu}}\right\}$.

Note: 1. Theorem 2 showed that the new upper bound is smaller than the old one in (6). 2. The equality holds for the old and new bounds if and only if $A_{0}=\sqrt{\frac{2 \mu \alpha_{2}}{1+\mu}}$, i.e. $\alpha_{2}=\frac{\mu}{2(1+\mu)}$, thus $E X^{2}=\mu^{2}$. This leads to a degenerated distribution with a point mass on a constant variable $X$. Hence the tight upper bound holds for any non-degenerated dNBUE distribution. For example, the uniform discrete random variable $X$ has either 0,1 or 2 with probability $\frac{1}{3}$ each, then $\mu=1$, and $\bar{F}(0)=\bar{G}(0)=1, \bar{F}(1)=\frac{2}{3}>\bar{G}(1)=\frac{1}{2}, \bar{F}(2)=\frac{1}{3}>\bar{G}(2)=\frac{1}{6}$, thus $X \in$ dNBUE. In addition $\beta_{2}=\frac{1}{2} E(X+2)(X+1)=$ $\frac{10}{3}, \alpha_{2}=1-\frac{\beta_{2}}{(1+\mu)^{2}}=\frac{1}{6}, A_{0}=\frac{-1+\sqrt{1+8 \alpha_{2}(1+\mu)^{2}}}{2(1+\mu)}=\frac{-1+\sqrt{\frac{19}{3}}}{4}=0.37915<\sqrt{\frac{2 \mu \alpha_{2}}{1+\mu}}=\sqrt{\frac{1}{6}}=0.4082$, and so the new bound $1-\exp \left\{-\frac{1+\mu}{\mu} A_{0}\right\}=0.5315$ is strictly smaller than the old bound $1-\exp \left\{-\sqrt{\frac{2(1+\mu) \alpha_{2}}{\mu}}\right\}=0.5580$.

\subsection{The dDMRL Class}

We now consider the approximation between $\mathrm{dDMRL}$ and the geometric. Notice that $\mathrm{dDMRL} \subset \mathrm{dNBUE}$ and $\mu(k)=$ $\frac{(1+\mu) \bar{G}(k)}{\bar{F}(k)}$ is decreasing in $k \in \mathscr{N}$, so $\mu(0)=1+\mu \geq \mu(k)=\frac{\sum_{j=k}^{\infty} \bar{F}(j)}{\bar{F}(k)}=1+\frac{\sum_{j=k+1}^{\infty} \bar{F}(j)}{\bar{F}(k)} \geq 1, k \in \mathscr{N}$. We denote $h(k)=$ $k-[1+\mu-\mu(k)]$. Here were the preliminary results in [12].

Lemma 9. Suppose $X \in d D M R L$ and $k \in \mathscr{N}$, then

(1) $0 \leq h(k) \leq k$;

(2) $A(i) \geq A(k)-\frac{k-i}{1+\mu} \bar{F}(k)$ for $h(k) \leq i \leq k$;

(3) $\sum_{i=k+1}^{\infty} A(i) \geq A(k)[\mu(k)-1]$;

(4) $\delta(k) \leq \bar{F}(k)\left\{1-\exp \left[-\left(1-\frac{\mu(k)-1}{\mu}\right)\right]\right\}$.

The the following result plays an essential role in our study to improve the upper bound.

Lemma 10. If $X \in d D M R L$, then $\sum_{i=0}^{k} A(i) \geq \frac{A(k)}{2}\left[\frac{(1+\mu) A(k)}{\bar{F}(k)}+1\right], k \in \mathscr{N}$.

Proof. Since $0 \leq h(k) \leq k$, we denote $h+\omega=h(k)=k-[1+\mu-\mu(k)]$ with $h \in \mathscr{N}, 0 \leq \omega<1$, thus $k-h=1+\mu-$ $\mu(k)+\omega=\frac{(1+\mu) A(k)}{\bar{F}(k)}+\omega$. By Lemma 9 with $k \geq 1$, we have

$$
\begin{aligned}
& \sum_{i=0}^{k} A(i) \geq \sum_{i=h+1}^{k} A(i) \geq \sum_{i=h+1}^{k}\left[A(k)-\frac{k-i}{1+\mu} \bar{F}(k)\right]=(k-h)\left[A(k)-\frac{k-h-1}{2(1+\mu)} \bar{F}(k)\right] \\
& =\left(\frac{(1+\mu) A(k)}{\bar{F}(k)}+\omega\right)\left[A(k)-\frac{\bar{F}(k)}{2(1+\mu)}\left(\frac{(1+\mu) A(k)}{\bar{F}(k)}+\omega-1\right)\right] \\
& =\frac{1}{2}\left[\frac{(1+\mu) A^{2}(k)}{\bar{F}(k)}+A(k)+\frac{\omega(1-\omega) \bar{F}(k)}{1+\mu}\right] \geq \frac{A(k)}{2}\left[\frac{(1+\mu) A(k)}{\bar{F}(k)}+1\right]
\end{aligned}
$$

The above inequality still holds for $k=0$ since $A(0)=0$. 
Then we obtain a smaller upper bound in the following.

Theorem 3. Suppose $X \in d D M R L$ and $Y \in G e o\left(\frac{1}{1+\mu}\right)$, then

$\Delta(X, Y) \leq 1-\exp \left\{-\frac{2(1+\mu) \alpha_{2}}{\mu}\right\}$

Proof.For $X \in \mathrm{dDMRL}$, by Lemmas 9 and 10, $A(k)=\bar{F}(k)-\bar{G}(k), \mu(k)=\frac{(1+\mu) \bar{G}(k)}{\bar{F}(k)}$, we have

$$
\begin{aligned}
& (1+\mu) \alpha_{2}=\sum_{i=0}^{\infty} A(i)=\sum_{i=0}^{k} A(i)+\sum_{i=k+1}^{\infty} A(i) \geq \frac{A(k)}{2}\left[\frac{(1+\mu) A(k)}{\bar{F}(k)}+1\right]+A(k)[\mu(k)-1] \\
& =\frac{A(k)}{2}\left[\frac{(1+\mu) A(k)}{\bar{F}(k)}+2 \mu(k)-1\right]=\frac{A(k)}{2}\left[\mu+\frac{(1+\mu) \bar{G}(k)}{\bar{F}(k)}\right]=\frac{A(k)}{2}\left[\mu+\frac{\sum_{j=k}^{\infty} \bar{F}(j)}{\bar{F}(k)}\right] \\
& =\frac{A(k)}{2}\left[1+\mu+\frac{\sum_{j=k+1}^{\infty} \bar{F}(j)}{\bar{F}(k)}\right] \geq \frac{(1+\mu) A(k)}{2}
\end{aligned}
$$

Hence $A(k) \leq 2 \alpha_{2}$, and thus $-\alpha_{2} \leq \delta(k) \leq 1-\exp \left\{-\frac{1+\mu}{\mu} A(k)\right\} \leq 1-\exp \left\{-\frac{2(1+\mu) \alpha_{2}}{\mu}\right\}$. In addition, $0 \leq \alpha_{2}<\frac{1}{2}$ from $\mathrm{dDMRL} \subset \mathrm{dNBUE} \subset \mathrm{dHNBUE}$. By elementary calculus, it is easy to show $\alpha_{2} \leq 1-\exp \left\{-\frac{2(1+\mu) \alpha_{2}}{\mu}\right\}$ in $0 \leq \alpha_{2}<\frac{1}{2}$, and hence we have

$$
\begin{aligned}
\Delta(X, Y) & =\sup _{k \in \mathscr{N}}|\delta(k)| \leq \max \left\{\alpha_{2}, 1-\exp \left\{-\frac{2(1+\mu) \alpha_{2}}{\mu}\right\}\right\} \\
& =1-\exp \left\{-\frac{2(1+\mu) \alpha_{2}}{\mu}\right\}
\end{aligned}
$$

Remarks: 1. The upper bound $1-\exp \left\{-\frac{2(1+\mu) \alpha_{2}}{\mu}\right\} \leq 1-\exp \left\{-2\left(\frac{1+\mu}{\mu}\right)^{2} \alpha_{2}\right\}$, an upper bound in [12]. 2. The equality holds if and only if $\alpha_{2}=0$, i.e. $X \sim G e o\left(\frac{1}{1+\mu}\right)$. Hence the tight upper bound holds for any non-geometric dDMRL distribution. For example, suppose a uniform discrete random variable $X$ has either 0,1 or 2 with probability $\frac{1}{3}$ each, then $\mu=1$, and $\bar{F}(0)=\bar{G}(0)=1, \bar{F}(1)=\frac{2}{3}, \bar{G}(1)=\frac{1}{2}, \bar{F}(2)=\frac{1}{3}, \bar{G}(2)=\frac{1}{6}, \mu(0)=\frac{2 \bar{G}(0)}{\bar{F}(0)}=2, \mu(1)=\frac{2 \bar{G}(1)}{\bar{F}(1)}=\frac{3}{2}, \mu(2)=\frac{2 \bar{G}(2)}{\bar{F}(2)}=$ 1, i.e. $\mu(k)$ is decreasing for $k=0,1,2$, thus $X \in \mathrm{dDMRL}$. Also $\alpha_{2}=\frac{1}{6}$, and so the new bound $1-\exp \left\{-\frac{2(1+\mu)}{\mu} \alpha_{2}\right\}=$ $1-\exp \left\{-\frac{2}{3}\right\}=0.4866<1-\exp \left\{-2\left(\frac{1+\mu}{\mu}\right)^{2} \alpha_{2}\right\}=1-\exp \left\{-\frac{4}{3}\right\}=0.7364$, the old bound.

Cheng and Ma [12] presented another upper bound $\Delta(X, Y) \leq 2\left(\frac{1+\mu}{\mu}\right)^{2}\left(1-e^{-1}\right) \alpha_{2}=U$, and we may also improve it in a similar way by using Lemmas 9 and 10 as follows. Since $(1+\mu) \alpha_{2}=\sum_{i=0}^{k} A(i)+\sum_{i=k+1}^{\infty} A(i) \geq \frac{A(k)}{2}\left[\frac{(1+\mu) A(k)}{\bar{F}(k)}+1\right]+$ $A(k)[\mu(k)-1]=\frac{A(k)}{2}[\mu+\mu(k)], 2 \alpha_{2} \geq A(k)\left[1+\frac{\mu(k)-1}{1+\mu}\right]$. By Lemma 9(4), we have

$$
\begin{aligned}
& \delta(k) \leq \frac{2 \alpha_{2} \bar{F}(k)}{A(k)\left[1+\frac{\mu(k)-1}{1+\mu}\right]}\left\{1-\exp \left[-\left(1-\frac{\mu(k)-1}{\mu}\right)\right]\right\} \\
& =\frac{2 \alpha_{2}}{\left[1+\frac{\mu(k)-1}{1+\mu}\right]\left[1-\frac{\mu(k)}{1+\mu}\right]}\left\{1-\exp \left[-\left(1-\frac{\mu(k)-1}{\mu}\right)\right]\right\} \\
& =\left(1-\frac{1}{\mu(k)+\mu}\right)\left(\frac{1+\mu}{\mu}\right)^{2} \frac{2 \alpha_{2}}{1-\left(\frac{\mu(k)-1}{\mu}\right)^{2}}\left\{1-\exp \left[-\left(1-\frac{\mu(k)-1}{\mu}\right)\right]\right\}
\end{aligned}
$$




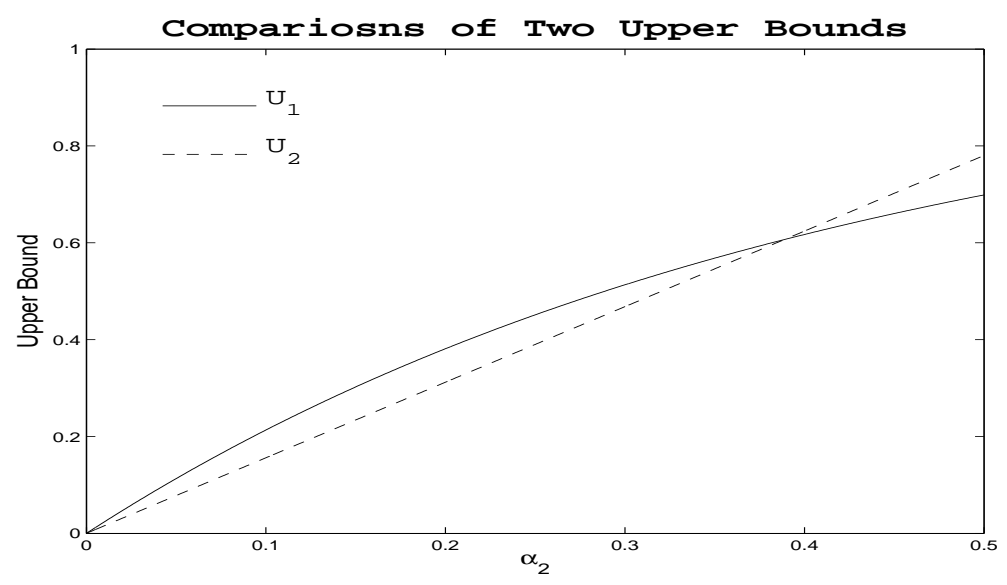

Fig. 1: Comparison of Two Upper Bounds

Let $t=1-\frac{\mu(k)-1}{\mu}$, for dDMRL, we know $1 \leq \mu(k) \leq \mu(0)=1+\mu, k \in \mathscr{N}$, and so $t \in[0,1]$. Since $g(t)=\frac{1-e^{-t}}{t(2-t)}$ is increasing in $t \in[0,1], g(t) \leq g(1)=1-e^{-1}$. Then

$-\alpha_{2} \leq \delta(k) \leq\left(1-\frac{1}{\mu(k)+\mu}\right) U \leq \frac{1+\mu}{2+\mu} U$

Also $\frac{1+\mu}{2+\mu} U=\frac{2(1+\mu)^{3}}{\mu^{2}(2+\mu)}\left(1-e^{-1}\right) \alpha_{2}>\alpha_{2}$, hence $\Delta(X, Y)=\sup _{k \in \mathscr{N}}|\delta(k)| \leq \frac{1+\mu}{2+\mu} U<U$.

We obtained two upper bounds for dDMRL with the geometric distribution. However, theoretically neither one is overall better. For example, a dDMRL distribution with $\mu=5$, Figure 1 displays the curves of the two bounds $U_{1}=$ $1-\exp \left\{-\frac{2(1+\mu) \alpha_{2}}{\mu}\right\}$ and $U_{2}=\frac{2(1+\mu)^{3}}{\mu^{2}(2+\mu)}\left(1-e^{-1}\right) \alpha_{2}$ in $0 \leq \alpha_{2}<\frac{1}{2}$, showing neither one is uniformly smaller than the other.

\section{Conclusions}

In this paper, we explored some characterizations of discrete life distributions, especially for dHNBUE, dHNWUE, dNBUE and dNWUE classes. We investigated the relations of those life distributions to the geometric distribution, and characterize the geometric distribution by an characteristic number within dHNBUE and dHNWUE classes. Furthermore, we provided the approximation through an upper bound of difference for dNBUE and dDMRL classes with geometric distribution, respectively. These upper bounds improved the ones given previously. Some future work is to address characteristics of other discrete life distributions and the relations among these and with the geometric distribution.

\section{Acknowledgment}

Wang and Xu's work was supported by the grants NSFC11141002, NSFC11041002, Shanghai Leading Academic Discipline Project (No. S3040), and Scientific Computing Key Laboratory of Shanghai Universities. Sha's work was partially supported by NSF-CMMI-0654417 and NIH-NIMHD 8G12MD007592.

\section{Appendix}

Proof of Lemma 3. 
Proof.For $X \in \mathrm{dHNBUE}$ with $\bar{G}(k) \leq\left(\frac{\mu}{1+\mu}\right)^{k}=\bar{F}_{g}(k)$, where $\bar{F}_{g}(k)$ is the survival function for $X_{g} \sim G e o\left(\frac{1}{1+\mu}\right)$, and expanding $(X+r)(X+r-1) \cdots(X+1)=\sum_{i=0}^{r} s(r, i)(X+1)^{i}=\sum_{i=0}^{r} s(r, i) \sum_{j=0}^{i}\left(\begin{array}{l}i \\ j\end{array}\right) X^{j}=\sum_{j=0}^{r} c(r, j) X^{j}$ with the unsigned Stirling number $s(r, j)[15]$ and $c(r, j)=\sum_{i=j}^{r} s(r, i)\left(\begin{array}{l}i \\ j\end{array}\right)$, we have by Lemma 1 ,

$$
\begin{aligned}
& \beta_{r}=\frac{1}{r !} E[(X+r)(X+r-1) \cdots(X+1)]=\frac{1}{r !} \sum_{j=0}^{r} c(r, j) E X^{j} \\
& =\frac{1}{r !} \sum_{j=0}^{r} c(r, j) \sum_{k=1}^{\infty}\left[k^{j}-(k-1)^{j}\right] \bar{F}(k)=\frac{1}{r !} \sum k=1^{\infty} \bar{F}(k) \sum_{j=0}^{r} c(r, j)\left[k^{j}-(k-1)^{j}\right] \\
& =\frac{1}{r !} \sum_{k=1}^{\infty} \bar{F}(k)\left[\sum_{j=0}^{r} c(r, j) k^{j}-\sum_{j=0}^{r} c(r, j)(k-1)^{j}\right] \\
& =\frac{1}{(r-1) !} \sum_{k=1}^{\infty} \bar{F}(k)[(k+r-1)(k+r-2) \cdots(k+1)] \\
& =\frac{1}{(r-1) !} \sum_{k=1}^{\infty} \bar{F}(k) \sum j=0^{r-1} c(r-1, j) k^{j}=\frac{1}{(r-1) !} \sum_{j=0}^{r-1} c(r-1, j) \sum_{k=1}^{\infty} k^{j} \bar{F}(k) \\
& =\frac{1+\mu}{(r-1) !} \sum_{j=0}^{r-1} c(r-1, j)\left[\sum k=1^{\infty} k^{j} \bar{G}(k)-\sum_{k=1}^{\infty} k^{j} \bar{G}(k+1)\right] \\
& =\frac{1+\mu}{(r-1) !} \sum_{j=0}^{r-1} c(r-1, j) \sum_{k=1}^{\infty}\left[k^{j}-(k-1)^{j}\right] \bar{G}(k) \\
& \leq \frac{1+\mu}{(r-1) !} \sum_{j=0}^{r-1} c(r-1, j) \sum_{k=1}^{\infty}\left[k^{j}-(k-1)^{j}\right] \bar{F}(k) \\
& =\frac{1+\mu}{(r-1) !} \sum_{j=0}^{r-1} c(r-1, j) E X_{g}^{j}=\frac{1+\mu}{(r-1) !} E\left[\left(X_{g}+r-1\right)\left(X_{g}+r-2\right) \cdots\left(X_{g}+1\right)\right] \\
& =(1+\mu) \beta_{r-1}^{g}=(1+\mu)^{r}=\beta_{r}^{g}
\end{aligned}
$$

so $\alpha_{r}=\left|1-\frac{\beta_{r}}{(1+\mu)^{r}}\right|=1-\frac{\beta_{r}}{(1+\mu)^{r}}$. For $X \in \mathrm{dHNWUE}$ with $\bar{G}(k) \geq\left(\frac{\mu}{1+\mu}\right)^{k}=\bar{F}_{g}(k)$, it is easily seen from the above that $\beta_{r} \geq \beta_{r}^{g}=(1+\mu)^{r}$, and so $\alpha_{r}=\frac{\beta_{r}}{(1+\mu)^{r}}-1$. In a similar manner, through the expansion expression $\mu_{(r)}=E[(X+r-$ 1) $(X+r-2) \cdots X]=\sum_{j=0}^{r} s(r, j) E X^{j}$, we will have $\mu_{(r)} \leq \mu_{(r)}^{g}$ for $X \in$ dHNBUE, and $\mu_{(r)} \geq \mu_{(r)}^{g}$ for $X \in$ dHNWUE.

Proof of Lemma 4.

Proof.(1) Since a similar approach can be applied to the case of dHNWUE, we just prove the result for $X \in$ dHNBUE. Note that $\beta_{r}=\beta_{r-1}+\frac{1}{r !} E[(X+r-1) \cdots(X+1) X]=\beta_{r-1}+\frac{\mu_{(r)}}{r !}$, then

$$
\begin{aligned}
\alpha_{r} & =1-\frac{\beta_{r}}{(1+\mu)^{r}}=1-\frac{\beta_{r-1}}{(1+\mu)^{r}}-\frac{\mu_{(r)}}{r !(1+\mu)^{r}} \\
& \geq 1-\frac{1-\alpha_{r-1}}{1+\mu}-\frac{\mu_{(r)}^{g}}{r !(1+\mu)^{r}} \\
& =\frac{\alpha_{r-1}}{1+\mu}+\frac{\mu}{1+\mu}-\frac{r ! \mu(1+\mu)^{r-1}}{r !(1+\mu)^{r}}=\frac{\alpha_{r-1}}{1+\mu}
\end{aligned}
$$

Also $E[(X+r)(X+r-1) \cdots(X+1)] \geq(E X+r)(E X+r-1) \cdots(E X+1)=(\mu+r)(\mu+r-1) \cdots(\mu+1)$ by Jensen's inequality for the convex function $f(x)=(x+r)(x+r-1) \cdots(x+1)$ in $x \geq 0, r \geq 1$, then for $X \in$ dHNBUE and $r \geq 2$, we have

$$
\begin{aligned}
\alpha_{r} & =1-\frac{\beta_{r}}{(1+\mu)^{r}}=1-\frac{E[(X+r)(X+r-1) \cdots(X+1)]}{r !(1+\mu)^{r}} \\
& \leq 1-\frac{(\mu+r)(\mu+r-1) \cdots(\mu+1)}{r !(1+\mu)^{r}}<1-\frac{1}{r !}
\end{aligned}
$$

Specifically, $\alpha_{2} \leq 1-\frac{(\mu+2)(\mu+1)}{2(1+\mu)^{2}}=\frac{\mu}{2(1+\mu)}<\frac{1}{2}$ and $\alpha_{3}<\frac{5}{6}$, which were the results in [12]. 
(2) For $X \in$ dNBUE with $\bar{G}(k) \leq \bar{F}(k), k \in \mathscr{N}$, from the derivation of Lemma 3, we have

$\beta_{r}=\frac{1+\mu}{(r-1) !} \sum_{j=0}^{r-1} C(r-1, j) \sum_{k=1}^{\infty}\left[k^{j}-(k-1)^{j}\right] \bar{G}(k)$

$\leq \frac{1+\mu}{(r-1) !} \sum_{j=0}^{r-1} C(r-1, j) \sum_{k=1}^{\infty}\left[k^{j}-(k-1)^{j}\right] \bar{F}(k)$

$=\frac{1+\mu}{(r-1) !} \sum_{j=0}^{r-1} C(r-1, j) E X^{j}=(1+\mu) \beta_{r-1}$

In addition, $X \in \mathrm{dNBUE} \subset \mathrm{dHNBUE}$, then

$\alpha_{r}=1-\frac{\beta_{r}}{(1+\mu)^{r}} \geq 1-\frac{(1+\mu) \beta_{r-1}}{(1+\mu)^{r}}=1-\frac{\beta_{r-1}}{(1+\mu)^{r-1}}=\alpha_{r-1}$

Alternatively, for $X \in \mathrm{dNWUE}$ with $\bar{G}(k) \geq \bar{F}(k), k \in \mathscr{N}$, we have $\beta_{r} \geq(1+\mu) \beta_{r-1}$, and so $\alpha_{r}=\frac{\beta_{r}}{(1+\mu)^{r}}-1 \geq \alpha_{r-1}$.

Proof of Lemma 8.

Proof. (1) First, by Lemma $4, \alpha_{2} \leq \frac{\mu}{2(1+\mu)}$, so it is easily seen that

$A_{0} \leq \sqrt{\frac{2 \mu \alpha_{2}}{1+\mu}} \leq \frac{\mu}{1+\mu}$

Next, we show $A \leq A_{0}$. Since $\bar{F}(0)=\bar{G}(0)=1$, then $A(0)=\bar{F}(0)-\bar{G}(0)=0$. Due to $\sum_{k=0}^{\infty} A(k)=(1+\mu) \alpha_{2}>0$, there exists $t$ such that $A(t)>0$ with $t \in \mathscr{N}_{+}$. Let $h(t)=t-(1+\mu) A(t)=h+\omega$, where $h$ and $\omega$ are the integer and fraction parts of $h(t)$, respectively. From Lemma 5, we know that $h \geq 0,0 \leq \omega<1$. Also since $A(t)>0$, then $t=h+\omega+(1+\mu) A(t)>h$ (i.e. $t \geq h+1)$. By Lemma 5(3), we have

$$
\begin{aligned}
& (1+\mu) \alpha_{2}=\sum_{k=0}^{\infty} A(k) \geq \sum_{k=h+1}^{t} A(k) \geq \sum_{k=h+1}^{t}\left[A(t)-\frac{t-k}{1+\mu}\right] \\
& =(t-h)\left[A(t)-\frac{t-h-1}{2(1+\mu)}\right]=[(1+\mu) A(t)+\omega]\left[A(t)-\frac{(1+\mu) A(t)+\omega-1}{2(1+\mu)}\right] \\
& =\frac{1}{2}\left[(1+\mu) A^{2}(t)+A(t)+\frac{\omega(1-\omega)}{1+\mu}\right] \geq \frac{1}{2}\left[(1+\mu) A^{2}(t)+A(t)\right]
\end{aligned}
$$

i.e. $(1+\mu) A^{2}(t)+A(t)-2 \alpha_{2}(1+\mu) \leq 0$. So that

$A(t) \leq A_{0}=\frac{-1+\sqrt{1+8 \alpha_{2}(1+\mu)^{2}}}{2(1+\mu)}$ for all $A(t)>0$

then $A=\sup _{k \in \mathscr{N}} A(k)=\sup _{t \in \mathscr{N}_{+}, A(t)>0}\{A(t)\} \leq A_{0}$.

(2) Actually, we have

$$
\begin{aligned}
& \alpha_{2} \leq 1-\exp \left\{-\frac{1+\mu}{\mu} A_{0}\right\} \Longleftrightarrow 1-\alpha_{2} \geq \exp \left\{-\frac{1+\mu}{\mu} A_{0}\right\} \\
& \Longleftrightarrow \log \left(1-\alpha_{2}\right) \geq-\frac{-1+\sqrt{1+8 \alpha_{2}(1+\mu)^{2}}}{2 \mu} \\
& \Longleftrightarrow\left[2 \alpha_{2}-\log ^{2}\left(1-\alpha_{2}\right)\right] \mu^{2}+\left[4 \alpha_{2}+\log \left(1-\alpha_{2}\right)\right] \mu+2 \alpha_{2} \geq 0
\end{aligned}
$$

Since $X \in \mathrm{dNBUE} \subset \mathrm{dHNBUE}$, from Lemma $4,0 \leq \alpha_{2}<\frac{1}{2}$, it is easily seen that $2 \alpha_{2}-\log ^{2}\left(1-\alpha_{2}\right) \geq 0$ and $4 \alpha_{2}+$ $\log \left(1-\alpha_{2}\right) \geq 0$. The lemma follows.

\section{References}

[1] Adams, G. and Watson, R. (1989). A discrete time parametric model for the analysis of failure time data. Australian Journal of Statistics. 31, 365-384.

[2] Bain, L.J. (1991). Statistical Analysis of Reliability and Life Testing Models. Marcel Dekker, New York.

[3] Best, D.J. and Rayner, J.C.W. (1989). Goodness of fit for the geometric distribution. Biometrical Journal, 31(3), 307-311. 
[4] Best, D.J. and Rayner J.C.W. (2003). Test of fit for the geometric distribution. Communications in Statistics: Simulation and Computation, 32(4), 1065-1078.

[5] Brown, M. and Ge, G. (1984). Exponential approximations for two classes of aging distributions. Annals of Probability, 12, $869-875$.

[6] Cheng, K. (1999). Life Distributions and Mathematical Theory of Reliability. Science Press.

[7] Cheng, K. and He, Z. (1993). Reliability bounds in NWUE class with known mean and variance. Journal of Systems Sciences and Complexity, 6, 145-149.

[8] Cheng, K. and He, Z. (1995). Reliability bounds in DFRA class with known mean and variance. Acta Mathematicae Applicatae Sinica (English Series), 11, 113-122.

[9] Cheng, K. and Lam, Y. (2001). Reliability bounds in HNBUE life distributions with known first two moments. European Journal of Operational Research, 132(1), 163-175.

[10] Cheng, K. and Lam, Y. (2002). Reliability bounds in NBUE life distributions. Naval Research Logistics, 49(8), $781-797$.

[11] Cheng, K. and Ma, B. (1989). On proximity between geometric and some discrete life distributions. Technical Report.

[12] Cheng, K. and Ma, B. (1991). Approximating distributions in discrete DMRL and NBUE classes. Journal of Systems Sciences and Complexity, 4, 264-274.

[13] Fang, K.T. and Xu, J.L. (1987). Statistical Distributions. Science Press.

[14] Ferguson, T.S. (1967). On characterizing distributions by properties of order statistics. Sankhya A, 29(3), 265-278.

[15] Graham, R.L., Knuth, D.E. and Patashnik, O. (1988). Concrete Mathematics. Addison-Wesley.

[16] Kullback, S. and Leibler, R.A. (1951). On Information and Sufficiency. Annals of Mathematical Statistics, 22(1), 79-86.

[17] Nanda, A.K. (2005). Discrete life distributions with decreasing reversed hazard. Sankhya, 67(1), 106-125.

[18] Stephens, M.A. (1974). EDF statistics for goodness of fit and some comparisons. Journal of the American Statistical Association, 69(347), 730-737.

[19] Xekalaki, E. (1983). Hazard functions and life distributions in discrete time. Communications in Statistics: Theory and Methods, 12, 2503-2509.

[20] Xu, X. and Fei, H. (2004). The geometric features of typical number for Geometric distribution and dHNBUE class. Technical Report, Shanghai Jiaotong University.

[21] Zhao, Q., Xiao, X., Wang, Z. and Lu, D. (2000). The duration information in a continuous speech recognition of applied research. Journal of Acoustics, 25(2), 175-181. 